\title{
LA ACTIVIDAD INVESTIGATIVA DE LOS DOCENTES UNIVERSITARIOS
}

Ana M. Arellano

(pp 54-68)

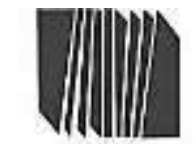

\section{Revista}

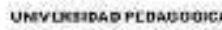
EOTH ME:MTAL LUESHTAO O
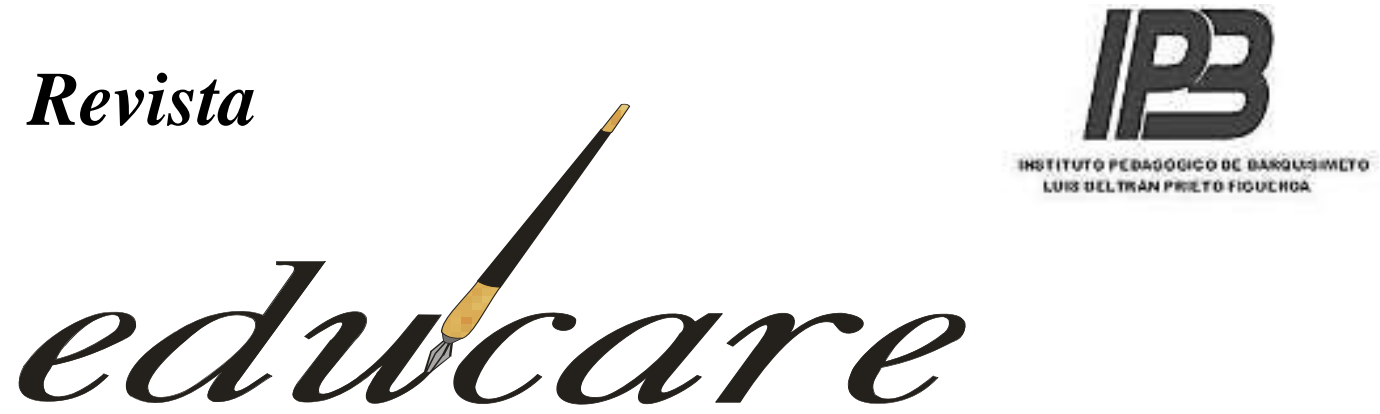
LUIS UCLTKAN PELCTO FIOUCHEA

Órgano Divulgativo de la Subdirección de Investigación y Postgrado del Instituto Pedagógico de Barquisimeto "Luis Beltrán Prieto

Figueroa"

Barquisimeto Estado Lara

EDICIÓN XX ANIVERSARIO

\section{LA ACTIVIDAD INVESTIGATIVA DE LOS DOCENTES UNIVERSITARIOS}

$$
\text { RESEARCH ACTIVITY OF UNIVERSITY TEACHERS }
$$

ISSN: 2244-7296

Autor

Ana M. Arellano

UPEL-IMPM 


\section{$\underline{\text { Ensayo }}$}

Recibido:08-04-2017

\section{RESUMEN}

Este ensayo aborda la importancia hacia la actividad investigativa de los docentes universitarios, visto como actitud y competencia profesional, estos no pueden ser unos simples repetidores de los conocimientos estáticos y acabados, sino por el contrario, deben ser críticos y con actitudes constantes de búsqueda del saber y mejoramiento; para ello resulta imperativo el ejercicio de la investigación como práctica permanente, con el apoyo del desarrollo de todas las competencias necesarias que faciliten dicho proceso. En el desarrollo se hace una definición de competencias, características, clasificación y considerando el criterio de Dalziel y Otros (2002), las competencias pueden dividirse en genéricas, técnicas y psicológicas, considerándose solo la primera, indicándose su conceptualización, de igual forma se hace referencia a los conocimientos y actitud del docente en el área de investigación, coinciden la mayoría de los autores citados en el componente actitud el cual es asumido como una disposición de aceptación o rechazo hacia la actividad investigativa, considerando tres componentes: cognitivo, afectivo y conductual.

Palabras clave: Docente universitario Investigación, Actitud, Competencias
Ana M. Arellano*

Aceptado:01-09-2017

\begin{abstract}
This essay addresses the importance of the research activity of the university professors, seen as attitude and professional competence, these cannot be simple repeaters of the static and finished knowledge, but on the contrary, they must be critical And with constant pursuit attitudes know and improve; To do this, it is essential to carry out the research as a permanent practice, with the support of the development of all the necessary competencies that facilitate this process. In the development a definition of competencies, characteristics, classification and considering the criterion of Dalziel and others (2002), the competencies can be divided into generic, technical and psychological, considering only the first one, which indicates its Conceptualization, in the same way that refers to the knowledge and attitude of the teacher in the area of research, the majority of the authors quoted coincide in the component attitude that is assumed as disposition of acceptance or rejection of the investigation Activity, considering three components: cognitive, affective and behavioural
\end{abstract}

Keywords: investigation, Transcompleja, School.

* Lcda. En Ciencias Politicas Esp. Gerencia de Recursos Humanos. MSC. Docencia para la Educacion Superior. Dra. Ciencias Educativas, es profesora contratada en la UNEFA y en la UPEL-IMPM Trujillo. Es miembro de la línea de Investigación Educativa "Familia y Sociedad", del núcleo de investigación "Ramón David Sánchez Palomares" en el área de docencia. anamaritza_a@hotmail.com 


\section{LA ACTIVIDAD INVESTIGATIVA DE LOS DOCENTES UNIVERSITARIOS \\ Ana M. Arellano \\ (рp 54-68)}

\section{INTRODUCCIÓN}

A nivel global, el impacto del desarrollo actual de la sociedad se ha convertido en un desafío inaplazable para la humanidad, implicando la necesidad de buscar nuevas formas de conocimiento y experiencias para ser eficiente, debido a que en este siglo XXI se vive en situaciones y supeditado con un entorno cada vez cambiante, competitivo, con estructuras organizacionales más esbeltas, complejas, sin olvidar como en los procesos sociales existe un aumento de la incertidumbre que en el pasado siglo.

Dada la importancia de la ciencia en el mundo, exige la reflexión acerca de muchos procesos de investigación, también a la gran era tecnológica la cual se viene desarrollando en los últimos tiempos, está exigiendo más de la ciencia para obtener resultados efectivos y exactos. Sin la ciencia nada de lo que ahora es utilizado estaría presente más sin embargo, todo tiene sus pros y sus contras. El gran desarrollo de la tecnología combinado con la ciencia muchas veces crea esos conflictos entre sociedades y creencias, conllevando a guerras y diferencias sociales.

Por una parte, la importancia de la investigación con relación a la sociedad, es ayudar a resolver problemas, actualmente se plantea la reeducación o el surgimiento de un nuevo modelo educativo como lo es la sociedad del conocimiento, en la cual la creación de conocimientos nuevos es una de las fuentes principales de la riqueza y del bienestar social. Esto es lo que haría que la sociedad actual sea realmente nueva desde el punto de vista económico, social y cultural, así como político, porque no siempre la fuente principal de bienestar y riqueza ha sido el conocimiento.

Sin embargo, la educación es y seguirá siendo la base del cambio de un país en todos sus niveles, estructuras. Es por ello se hace indispensable que ésta vaya hacia la búsqueda de la perfección, la solidez, excelencia teniendo como pilar del éxito, la efectividad de todos los componentes involucrados en el proceso educativo, entre ellos las personas que la llevan a cabo. En tal sentido, la educación venezolana requiere de profundas y positivas transformaciones que aseguren la formación del ciudadano de hoy y del mañana, capaz de contribuir al desarrollo del país y de participar plenamente en la vida democrática. Una de

Volumen $21 N^{\circ} 3$ Septiembre -Diciembre 2017. ISSN: 2244-7296 P 56 
las medidas para lograrlo la constituye la toma de conciencia por parte del docente, del rol que debe desempeñar en la educación como investigador, para así, edificar, nutrir las bases que sustentan su acción pedagógica.

Ahora bien las instituciones de educación universitaria, requieren hoy día un personal académico altamente competente para el desempeño de sus funciones capaces de responder a los retos que plantea el ámbito educativo y social. Por lo tanto, Romero y González (2009) indican como el docente universitario para llevar a cabo sus diferentes funciones de docencia, investigación y extensión, deberá actuar desde su condición humana compleja, haciendo uso de sus talentos innatos y de aquellos que deberá desarrollar involucrándose en procesos de formación que le conlleve a ello.

Se hace indispensable, para trabajar en las diferentes áreas académicas, el docente reúna una serie de actitudes y competencias profesionales, las cuales le permitan, tanto a él como a los estudiantes desempeñarse individual y colectivamente, en diferentes actividades y escenarios. Todo docente comprometido con su labor de enseñar y promover el aprendizaje debe reflexionar sobre su quehacer permanentemente, proponiendo innovaciones que tengan como base la investigación haciendo uso de las teorías más recientes o sencillamente repensándolas. En el ejercicio docente actual, sobre todo en el universitario, ya no es suficiente desempeñarse como dador de conocimientos ni expositor de clases.

El docente debe estar en constante búsqueda de conocimientos por medio de la investigación, considerando las estrategias de aprendizaje como hipótesis de acción, examinándolas y comprobando su eficiencia con el fin de modificarlas y sustituirlas. El desarrollo del rol de investigador requiere de un profesional dinámico, creador, con gran curiosidad intelectual y ciertas habilidades que le faciliten adaptarse al cambio. Se requiere un profesional creativo, es decir, capaz de establecer múltiples conexiones entre diferentes elementos que pueden configurar un sistema y, a través de un razonamiento lógico, formular nuevas hipótesis que conduzcan a la creación de nuevos conocimientos.

En este orden de ideas, la vinculación entre la actividad docente e investigación en el sector universitario resulta un tema de interés, razón por la cual ha sido abordado por Clemenza, Ferrer y Araujo (2006), concluyendo como se debe de redefinir las funciones básicas docentes para lograr dicha vinculación, aprovechando el potencial de los docentes. 
Esto ha representado un tema neurálgico e inquietante, tomando en cuenta la responsabilidad y razón de ser de las universidades ante las diversas problemáticas del entorno. Sin duda, las universidades representan la principal vía para el desarrollo potencial de la investigación al servicio de la sociedad en general; a través de la producción de conocimiento con alta utilidad e impacto colectivo.

En síntesis la investigación a nivel universitario representa un factor de gran importancia en la búsqueda del saber, de nuevos conocimientos y prácticas de gestión en las diferentes ramas, tanto naturales como humanísticas, para el discernimiento más profundo de la realidad, siendo el eje de toda institución universitaria en la promoción de saberes, pues es el instrumento por excelencia a través del cual se puede reflejar la realidad de un país, de la comunidad y de la misma institución que la promueve, también es la vía para plantear o solucionar alternativas a los problemas socioeducativos, políticos, económicos y culturales que emerja del contexto de desarrollo del docente y su compromiso con la sociedad en la cual está inmerso.

Esta situación tal vez, de la poca importancia de la investigación en el ámbito universitario sea producto de la prevalencia otorgada a la actividad docente sobre ella. Según Chacín y Otros (2010) la investigación en el entorno universitario ha sido caracterizada como desarticulada, individualista, desvinculada, sin respuesta a las exigencias sociales, con poca preocupación por su producción y sin promoción para su aprovechamiento; desvirtuándose de esta manera, su concepción y una de sus funciones primordiales como es la colaboración activa en el proceso de desarrollo de las comunidades y del país. Esto

En este orden de ideas, Mascherin (2010:24), indica como una actitud es una forma característica del individuo de responder a un objeto o situación. Se basa en la experiencia previa y en su interpretación y conduce a determinado comportamiento o determinadas opiniones. La autora plantea que los individuos presentan esquemas conductuales predeterminados en función de sus experiencias previas, lo que incide a manifestar ciertas conductas según sea el caso. Las actitudes siempre están presentes y se hacen aparentes a través del comportamiento frente una determinada situación, donde las mismas se refuerzan con el dominio efectivo, pero muchas veces se adquieren por el aprendizaje de procesos

Volumen $21 N^{\circ} 3$ Septiembre -Diciembre 2017. ISSN: 2244-7296 P 58 
sutiles, las actitudes pueden ser racionales o irracionales y se basan en información limitada.

Ahora bien, el desarrollo de la investigación es una ocupación de compromiso del docente con y para la sociedad, la universidad lo que constituye un imperativo, ya que las consecuencias de dicha actividad crean un aporte favorable a los problemas emergen de la sociedad. De igual forma, los productos de la investigación y la praxis cotidiana de la misma por parte de los docentes investigadores, significa una contribución reveladora para el mejoramiento de la calidad de la docencia y de desarrollo profesional en tiempos de cambio

Por todo lo planteado, podría inferir que no se han desarrollado en los docentes, como guía en las universidades, las actitudes y competencias necesarias las cuales favorezcan la investigación permanente, pareciera que ello va de la mano con la prevalencia de una actitud de rechazo o evasión hacia la actividad investigativa y no la de aceptación en función de favorecer la producción intelectual permanente. En líneas generales, el cambio social demanda que los docentes se animen, sean creativos, capaces de asimilar y adaptarse a los nuevos cambios, lo cual debe conducir a reformular el papel del docente, así como el modo de definir sus tareas y funciones.

Es importante destacar como la docencia y la investigación es una unidad monolítica que representa un factor de importancia en la búsqueda del saber y de nuevos conocimientos en las diferentes ramas, tanto naturales como humanísticas. Las universidades y su colectivo docente tienen el reto de generar y promover el conocimiento, porque este es el instrumento por excelencia a través del cual se puede reflejar la realidad de un país, de la comunidad y de la misma institución. Desde esta perspectiva, se considera que el docente en ejercicio debe participar activamente en la investigación de su propia práctica, lo que implica que docencia e investigación deben estar estrechamente unidas y ser asumidas por el educador como una actividad intrínseca del cómo ser socializador y productor de conocimiento.

Sin embargo, la formación para la investigación es un proceso continuo de acciones orientadas a favorecer la apropiación y desarrollo de los conocimientos, habilidades y actitudes necesarios para que los docentes puedan desempeñar con éxito actividades productivas asociadas a la investigación científica, el desarrollo científico - tecnológico y la 
innovación, ya sea en el sector académico o en el productivo. Es importante destacar que la actividad investigativa tiene un conjunto de principios contemplados como: universalidad, democracia, innovación y pertinencia social, equidad y calidad; estos principios revelan el compromiso que tienen los investigadores docentes frente a la sociedad encargados de fortalecer la formación integral del investigador en el ámbito socioeducativo.

En opinión de Hernández (2010), para perfeccionar el proceso de formación del docente investigador universitario es necesario aplicar mejoras significativas en las investigaciones a nivel institucional tanto académicas como financieras esto con el objetivo de estimular y propiciar los ejes que involucren recientes formas de realizar investigaciones de calidad y garantizar diversidad de perspectivas en el contexto académico, científico y ético.

Para las universidades, resulta necesario identificar las competencias y aptitudes que actualmente poseen sus docentes, necesarias para cumplir con su rol de investigador a cabalidad, pudiendo conectar así el saber científico a la solución de los problemas que el país, en primera instancia, le demanda. Es necesario saber qué competencias y aptitudes se tienen y cuáles no, para desarrollarlas y así poder avanzar de manera favorable en la actitud que se tiene frente a tan magna responsabilidad.

Lo anteriormente planteado sugiere, por un lado, la necesidad de determinar sus competencias para la investigación; así como la actitud o disposición hacia dicho objeto actitudinal, involucrando a lo anterior, la descripción de sus creencias, emociones y conductas al respecto; y establecer su posible relación, para definir así cada uno de los aspectos que favorezcan la intención de la investigación, como paso inicial hacia la búsqueda de conclusiones útiles para la toma de decisiones gerenciales que impulsen verdaderamente y de manera acertada, la producción intelectual de los docentes universitarios.

\section{COMPETENCIAS PARA LA INVESTIGACIÓN}

Según Buzo (2010) una síntesis de este concepto, después de analizar un elevado número de definiciones de la competencia, elaboradas por una gran diversidad de autores e instancias nacionales e internacionales, tanto educativas como profesionales, de la cual

Volumen $21 N^{\circ} 3$ Septiembre-Diciembre 2017. ISSN: 2244-7296 P 60 
extraer algunos elementos comunes a tener en cuenta: a) en el concepto de competencia se integra el saber, el saber hacer y el saber ser; b) se constituye de conocimientos, habilidades $\mathrm{y}$ actitudes que producen resultados tangibles, y su medida nos informa del grado de dominio conseguido; c) tiene relación con la acción: se desarrolla, se actualiza en la acción; d) Está vinculada a un contexto, a una situación dada; e) facilita la resolución eficaz de situaciones laborales conocidas o inédita; y f) es educable.

De acuerdo a la síntesis del autor antes citado la definición del perfil competencial del profesor universitario, tal como es entendida por el investigador, no puede separarse de las dos principales funciones profesionales que debe asumir (docencia e investigación) ni de los ya mencionados escenarios donde esta se desarrolla (contexto social, contexto institucional y micro contexto).

En este sentido, el no contar el docente universitario con las competencias requeridas para llevar a cabo su rol de investigador, puede de cierta manera predisponerle a actuar de manera desfavorable hacia este tipo de función. De allí que sea necesario determinar qué competencias poseen para posteriormente definir hasta qué punto las debilidades en sus competencias pudieran estar afectando su actitud de manera negativa; o si por el contrario, poseer las competencias requeridas le predispone de manera favorable ante la actividad investigativa.

Por su parte, las competencias afectan la puesta en práctica integrada de aptitudes, rasgos de personalidad y también de conocimientos adquiridos para cumplir una misión en una organización. De allí que puede no puede decirse que las competencias no están en relación con las aptitudes y los rasgos de personalidad. Por el contrario, constituyen una categoría específica de características individuales relacionadas a su vez, con los valores y conocimientos adquiridos. Las competencias no pueden desarrollarse si las aptitudes requeridas no están presentes, pero no se reducen a una aptitud. Las competencias permiten sobrepasar los límites del funcionamiento cognitivo.

En efecto, permite actualizar sistemas de información y utilizarlos sin tener que concentrar en ellos nuestra atención. Están ancladas en comportamientos observables en el ejercicio de un oficio o de un empleo y que se traducen en comportamientos que contribuyen al éxito profesional en el mismo. Son fruto de la experiencia, pero que se

Volumen $21 N^{\circ} 3$ Septiembre-Diciembre 2017. ISSN: 2244-7296 P 61 
adquieren a condición de que estén presentes las aptitudes y los rasgos de personalidad. (aptitudes y rasgos de personalidad + experiencias = competencia). La importancia de las aptitudes intelectuales y capacidades cognoscitivas es vital en las competencias.

Resumiendo, puede decirse que las competencias constituyen un vínculo entre la ejecución de una tarea y los comportamientos puestos en práctica, por una parte, y las cualidades individuales necesarias para comportarse de manera satisfactoria, por otra. Para Muñoz, Quintero y Munévar (2005), el término competencia proviene del latín competens, competentis (Moliner, 1998), el que tiene aptitud legal o autoridad para resolver cierto asunto o también el que conoce, es experto o apto en cierta ciencia o materia. Se puede entender por competencia "el conjunto de conocimientos, habilidades y actitudes que se aplican en el desempeño de una función productiva o académica”. (p. 15)

En este sentido, Tobón (2007), define las competencias como "procesos complejos de desempeño con idoneidad en determinados contextos, teniendo como base la responsabilidad" (p.45). De igual forma el mismo autor plantea (2007), que la mayoría de las definiciones expuestas sobre competencias las abordan en términos de atributos (por ejemplo conocimientos y habilidades), sin especificar si son acciones, actuaciones o estructuras implícitas. Se sale únicamente de este ámbito, la concepción de Lévy-Leboyer (ob. Cit), quien las plantea en términos de comportamientos que algunas personas dominan mejor que otras. Esta concepción refiere al modelo de competencias propio del conductismo laboral.

De acuerdo con los planteamientos del autor, las competencias implican, por un lado las actividades profesionales y por el otro, aprender a analizar y resolver problemas; aspecto que implica un enfoque investigativo, muy pertinente para esta investigación. Una competencia es entonces lo que hace que la persona sea, valga la redundancia, competente para realizar un trabajo o una actividad y exitoso en la misma, lo que puede significar la conjunción de conocimientos, habilidades, disposiciones y conductas específicas.

En sintonía con lo anterior, está el concepto de Maldonado (2002,) quien define a las competencias como "un conjunto de actitudes, valores, conocimientos y habilidades (personales, interpersonales, profesionales y organizacionales) que faculta a las personas para desempeñarse apropiadamente frente a la vida y el trabajo". Al hablar de competencias

Volumen $21 N^{\circ} 3$ Septiembre-Diciembre 2017. ISSN: 2244-7296 P 62 
personales y profesionales, se hace referencia a la aptitud, capacidad, idoneidad, habilidad, destreza y suficiencia en el desempeño de una función. Lo que interesa es lo que el individuo real y efectivamente "sabe hacer", independientemente de dónde y cómo lo haya aprendido.

Los planteamientos del autor citado, son muy pertinentes para esta investigación, tomando en cuenta que es propósito del autor, centrarse en los conocimientos y habilidades, como eje central de las competencias, dejando de lado los aspectos de orden estrictamente psicológico (rasgos de personalidad, por ejemplo), a los cuales hacen alusión de manera amplia, otros autores. Gerhard Bunk (citado por Maldonado 2002) define las competencias, como los conocimientos, destrezas y aptitudes que poseen los sujetos para ejercer una profesión, resolviendo los problemas cotidianos internos y externos a la organización. Por su parte, para Ruiz (2006) una competencia "es un constructo psicológico que ha sido usado para describir la habilidad que tiene un individuo para tomar iniciativas y actuar sobre su ambiente" (p. 69).

De la revisión conceptual realizada por Inciarte y González (2009), se consideró realizar una definición de las competencias, que pudiera considerar el docente, para reflexionar sobre la mediación en las áreas académicas como la investigación (p.52). El listado de competencias que se propone, representa un análisis de la manera como atender, la mediación del aprendizaje en las áreas de la investigación educativa y la evaluación de los aprendizajes, ya que de esa intervención, dependerá, la formación profesional integral, el docente deberá a partir de su acción en el aula, fomentar en el estudiante, el desarrollo de habilidades y destrezas para investigar y evaluar. Al respecto se definen las competencias en los siguientes párrafos:

Según señalan Dalziel y col (2002), las competencias personifican habilidades propias de los individuos, las cuales son relativamente permanentes, puesto que son sensibles al cambio, proveniente del entrenamiento sistemático. Asimismo, las competencias se encuentran caracterizadas por su esencialidad, es decir, por el hecho que no se puede obtener eficiencia sin su presencia. Por su parte, Escamilla (2008) afirma que entre las características de las competencias figuran las siguientes: evolución y educabilidad, 
complejidad, integración, idoneidad, adaptación al contexto, reflexión, creatividad, innovación y finalmente responsabilidad.

\section{ACTITUD DEL DOCENTE UNIVERSITARIO}

La actitud, como constructo teórico, ha sido abordada ampliamente por varios autores y en distintos momentos. Sus orígenes se encuentran en los aportes realizados por la Psicología Social. En este sentido, se esbozarán de manera sucinta, las principales conceptualizaciones en el marco de diferentes enfoques, para posteriormente definir el que servirá de sustento teórico central en esta investigación.

Una de las definiciones más usuales es la de Rosenberg y Hovland (1960, c.p. Hewstone y otros, 1994), quienes definen a las actitudes como "predisposiciones a responder a alguna clase de estímulos con ciertas clases de respuesta” (p.150). Estas respuestas son de tipo afectivas (concernientes a sentimientos evaluativos de agrado o desagrado), cognitivas (concernientes a creencias, opiniones e ideas acerca del objeto de actitud) y cognitivas/conductuales (concernientes a intenciones conductuales o tendencias de acción).

En este concepto se contempla la actitud como un constructo hipotético que media entre lo observable, estímulos y subsiguiente conducta. En este concepto puede enmarcarse la actitud hacia la actividad investigativa. La investigación como conducta en el docente universitario está estrechamente ligada a los conocimientos y opiniones; así como a sentimientos de agrado o desagrado ante todos los aspectos en ella implícitos. En este sentido, resulta interesante conocer, en primer término, qué conocimientos y opiniones tienen hacia la actividad investigativa (elemento cognoscitivo); lo que sienten sobre la misma (elemento afectivo) y las conductas, tanto a favor como en contra que despliegan en torno a esta parte de su labor universitaria (elemento conductual).

Define Myers (2005) a la actitud como "una reacción evaluativo, favorable o desfavorable, hacia algo o alguien, que se manifiesta en las propias creencias, sentimientos o en la intención del comportamiento" (p.223). Este concepto, de data más reciente, es congruente con los planteamientos originales de Rosenberg, Hovland y Allport. La actitud para Fishbein y Ajzen (2002), es "un juicio bipolar de un objeto...esencialmente subjetivo,

Volumen $21 N^{\circ} 3$ Septiembre -Diciembre 2017. ISSN: 2244-7296 P 64 
que se desplaza entre dos polos, uno positivo o favorable y otro negativo o desfavorable". (p.20).

En función de esto y siguiendo a los autores, se puede decir que el docente universitario de la Aldea se desplaza entre dos polos en su actitud hacia la actividad docente, en este caso particular, depende de sus creencias para organizar cognitivamente la carga afectiva a favor o en contra y actuar, en consecuencia, de acuerdo a sus creencias.

Congruentes con los anteriores planteamientos teóricos; Worchel, Cooper, Goethals y Olson (2002) definen la actitud como "un juicio evaluativo (bueno o malo) de un objetivo (...) representa la propensión favorable o negativa del individuo hacia el objetivo". (p. 126). Ahora bien, Sánchez (2001) desarrolló un concepto de actitud mucho más cercano al objeto actitudinal de este estudio (la investigación). La autora definió como actitud científica a la disposición o tendencia sistemática a comportarse como investigador que tiene el docente universitario, siempre que ocurran determinadas situaciones (lo externo), en correspondencia con la constitución de un conjunto de rasgos que engloban unos valores, información, creencias y habilidades, en el individuo (lo interno).

Todos los autores coinciden en plantear que es, sin duda, un estado interno y que se pueden realizar manifestaciones a través de respuestas observables que se han agrupado en tres categorías: cognitiva, afectiva y conductual. Los conceptos expuestos, permiten concluir como la actitud no es más que estados o posturas de la persona los cuales pueden ser aprendidas y surgen en base a la experiencia con el medio ambiente, situación específica u objeto. Las respuestas a emitir pueden estar condicionadas por las creencias, sentimientos y el conocimiento previo de la persona de ese objeto actitudinal.

Dicho de otra manera, es una predisposición el cual existe en el sujeto adquirida por el aprendizaje que impulsa al individuo a comportarse a favor o en contra de un objeto social definido, (en este caso, la actividad investigativa) de una forma determinada; y en dicha situación se establece un estado mental integrado por el componente cognitivo, afectivo y comportamental. Para la presente investigación, gran parte de los autores manejados, coinciden en los componentes de la actitud es asumida como una disposición de aceptación o rechazo hacia la actividad investigativa, considerando los tres componentes: cognitivo, afectivo y conductual (lo interno en el docente universitario) 


\section{LA ACTIVIDAD INVESTIGATIVA DE LOS DOCENTES UNIVERSITARIOS \\ Ana M. Arellano \\ (рp 54-68)}

Según Aldana \& Joya (2011), los componentes cognitivo, afectivo y conductual que integran las actitudes ejercen mutua influencia hacia un estado de armonía, y cualquier cambio que se registre en uno de estos tres componentes modificará a los otros dos, puesto que todo el sistema se altera cuando alguno de sus componentes lo hace. Así, una nueva experiencia o una nueva conducta emitida en cumplimiento de determinadas normas sociales u otro aspecto, pueden crear un estado de incongruencia entre los tres componentes actitudinales, dando lugar a un cambio de actitud.

\section{CONSIDERACIONES FINALES}

Como aporte este articulo acrecentará las actitudes hacia la investigación científica la cual es como una organización duradera y persistente de creencias hacia la misma, por parte de un colectivo; que este caso es la comunidad académica universitaria, en la que intervienen dimensiones afectivas, cognitivas y conductuales. La dimensión afectiva se manifiesta por sentimientos de agrado o desagrado y se expresan en términos de simpatíaantipatía hacia la investigación científica. La dimensión cognoscitiva representa el conocimiento consciente o pensamiento, dentro de ciertos límites de certeza, tienen de lo que es verdadero o falso, malo o bueno, deseable o indeseable, con respecto a la investigación científica. La dimensión conductual entendida como una acción o como predisposición a la acción relacionada con la investigación científica.

Por lo anterior, se debe de inducir en el docente la necesidad de actualización permanente y educación continua en el área de investigación; a través de la lectura de textos de metodología, cursos y postgrados en el área. De igual forma estimular como política universitaria, la adscripción de los docentes a centros de investigación y la ejecución de proyectos; dando amplia información sobre las líneas de investigación que existen dentro de la universidad. Se sugiere, además, definir incentivos académicos y económicos que coadyuven en tal sentido. 


\section{LA ACTIVIDAD INVESTIGATIVA DE LOS DOCENTES UNIVERSITARIOS \\ Ana M. Arellano \\ (рp 54-68)}

\section{REFERENCIAS}

Alanís, A. (2004) Saber y saber hacer en la investigación educativa. En Revista Cándidus. Enero-Marzo

Aldana, G. M. (2011). La formación en investigación, formación para la vida. En: J. C.

Arboleda (Ed.)Tomo 2 Colección Pedagogía Iberoamericana. Lenguaje, formación investigativa e integral. Colombia: Redipe

Aldana, G.M. \& Joya, N. S. (2011). Actitudes hacia la investigación. Tábula Rasa, No. 14, enero-junio.

Alcántara, José. (1998). Cómo educar las actitudes. CEAC. Barcelona, España.

Alles, Martha. (2005). Diccionario de comportamientos. Gestión por competencias. Granica. Argentina.

Alles, Martha. (2006). Desempeño por competencias. Evaluación de 360. Granica. Argentina

Carreras, J. y Perrenoud, P. (2005).El debat sobre les competències en l'ensenyament universitari. Barcelona: ICEUB. Disponible en:http://www.ub.edu/ice/universitat/quad erns.htm

Cifuentes, P., Alcalá, M.J. y Blázquez, M.R. (2005). Rol de profesorado en el EEES. XI Congreso de Formación del profesorado. Segovia, 17, 18 y 19 de Febrero.

Dalziel, M.; CUBEIRO, J. Y FERNÁNDEZ, G. (2002). Las Competencias Clave para Una Gestión de Recursos Humanos. Madrid: Ediciones Deusto.

Echeverría, B. (2002). Gestión de la competencia de acción profesional. Revista de Investigación Educativa, 20(1), 7-43.

Lévy-Leboyer, C. (2003). Gestión de las competencias.. Gestión 2000. Barcelona, España.

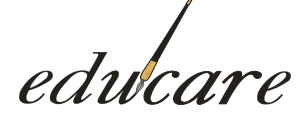

Volumen $21 N^{\circ} 3$ Septiembre -Diciembre 2017. ISSN: 2244-7296 P 67 


\section{LA ACTIVIDAD INVESTIGATIVA DE LOS DOCENTES UNIVERSITARIOS \\ Ana M. Arellano \\ (pp 54-68)}

Ley de Universidades (Gaceta Oficial No. 1429, Extraordinario de 8 de septiembre de 1970).

Maldonado, M. (2006) Competencias: Método y Genealogía. Ecoe Ediciones. Bogotá, Colombia

Maldonado, M. (2002) Las Competencias: Una opción de vida. Ecoe Ediciones. Bogotá, Colombia

Marquès, P. (2007) Los docentes: funciones, roles, competencias necesarias, formación. Disponible en: http://dewey.uab.es/pmarques/docentes.htm

Muñoz, J.; Quintero, J.; Munévar, R. (2005). Cómo desarrollar competencias investigativas en educación. Magisterio. 3ra. Edic. Colombia.

Romero I y González N. (2009). Competencias del docente de educación superior como mediador en los procesos de investigación y evaluación de los aprendizajes Omnia, Vol. 15, Núm. 2, mayo-agosto, pp. 39-55 Universidad del Zulia Venezuela

Sevillano G, (2005). Didáctica en el Siglo XXI. Ejes en el Aprendizaje y Enseñanza de Calidad. Editorial Mc Graw Hill. España

Tobón, S. (2007). Competencias en la educación superior. Edoe Bogotá, Colombia.

Tobón, S. (2007). Formación basada en competencias. Ecoe. Bogotá, Colombia.

Tobón, S.; Rial, A.; Carretero Díaz, M.; García, J. (2006). Competencias, calidad y educación superior. Magisterio. Bogotá, Colombia. 